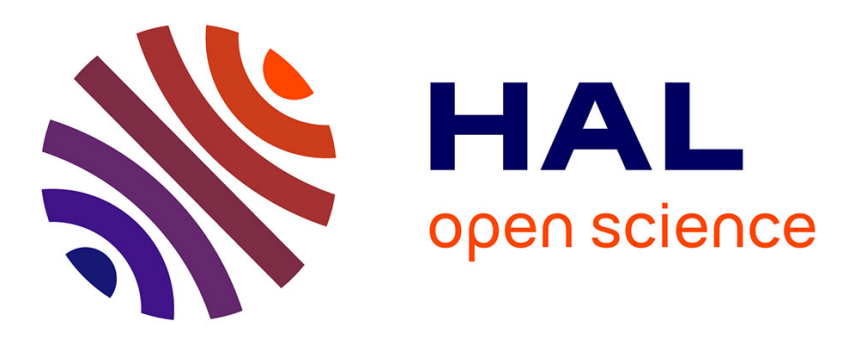

\title{
Expert-validated CSF segmentation of MNI atlas enhances accuracy of virtual glioma growth patterns
}

A Amelot, E Stretton, Hervé Delingette, Nicholas Ayache, S Froelich, E Mandonnet

\section{- To cite this version:}

A Amelot, E Stretton, Hervé Delingette, Nicholas Ayache, S Froelich, et al.. Expert-validated CSF segmentation of MNI atlas enhances accuracy of virtual glioma growth patterns. Journal of NeuroOncology, 2015, 121 (2), pp.381-387. 10.1007/s11060-014-1645-5 . hal-01081410

\section{HAL Id: hal-01081410 https://hal.inria.fr/hal-01081410}

Submitted on 7 Nov 2014

HAL is a multi-disciplinary open access archive for the deposit and dissemination of scientific research documents, whether they are published or not. The documents may come from teaching and research institutions in France or abroad, or from public or private research centers.
L'archive ouverte pluridisciplinaire HAL, est destinée au dépôt et à la diffusion de documents scientifiques de niveau recherche, publiés ou non, émanant des établissements d'enseignement et de recherche français ou étrangers, des laboratoires publics ou privés. 


\title{
Expert-validated CSF segmentation of MNI atlas enhances accuracy of virtual glioma growth patterns
}

\author{
A. Amelot ${ }^{1}$, E. Stretton ${ }^{2}$, H. Delingette ${ }^{2}$, N. Ayache ${ }^{2}$, S. Froelich ${ }^{1}$, \\ E. Mandonnet ${ }^{1,3,4}$ \\ ${ }^{1}$ Hôpital Lariboisière, Neurosurgery Department, Paris, France, \\ ${ }^{2}$ Asclepios Research Project, INRIA, Sophia Antipolis, France, \\ ${ }^{3}$ Université Paris 7, France, \\ ${ }^{4}$ IMNC, UMR 8165, Orsay, France
}

\begin{abstract}
Biomathematical modeling of glioma growth has been developed to optimize treatments delivery and to evaluate their efficacy. Simulations currently make use of anatomical knowledge from standard MRI atlases. For example, cerebrospinal fluid (CSF) spaces are obtained by automatic thresholding of the MNI atlas, leading to an approximate representation of real anatomy. To correct such inaccuracies, an expertrevised CSF segmentation map of the MNI atlas was built. Several virtual glioma growth patterns of different locations were generated, with and without using the expert-revised version of the MNI atlas. The adequacy between virtual and radiologically observed growth patterns was clearly higher when simulations were based on the expert-revised atlas. This work emphasizes the need for close collaboration between clinicians and researchers in the field of brain tumor modeling.
\end{abstract}

\section{Introduction}

Gliomas are brain tumors that remain incurable despite recent advances in treatments that combine surgery, chemotherapy and radiation therapy. Their diffuse behavior, resulting in an invasion of normal appearing parenchyma peripheral to the bulk lesion, is a major cause of treatment failure.

Biomathematical modeling of these tumors has been developed over the past two decades. The hope is that in silico tumor growth models could help to optimize treatments delivery $[2,5,23,24]$ and to evaluate their efficacy $[34,18$, $22]$. Such clinical applications require patient-specific inputs, obtained for example from pre-treatment sequential MRIs. Simulations are either performed in the patient MRI space [27] onto which anatomical knowledge of atlases can be matched (to obtain brain parenchyma segmentation and create white and gray matter segmentations), or in a reference space - usually the MNI atlas [9] - onto which all patient images are registered. Hence accurate anatomical atlases are needed in both cases.

\footnotetext{
$\overline{1}$ corresponding author: mandonnet@mac.com.
} 
Advances have been made in integrating a more precise anatomy in the atlas. Whereas the very first templates in the nineties were built from a 2D CT scan [35, $6,33]$, just outlining the brain surface and the ventricles, more recent works are based on 3D-MRI atlases of CSF, grey matter and white matter [28], and eventually including detailed white matter architecture via DTI sequences $[12,3,27$, 26]. CSF spaces are of utmost importance for achieving realistic glioma growth patterns, as they constitute anatomical barriers that cannot be crossed by tumor cells (a statement that is implemented in the model by a no flux boundary condition). The segmentation of CSF delineation is commonly obtained by an automated thresholding of the atlas, without any expert validation of its anatomical accuracy. For example, it has been previously shown that current templates contain unphysiological bridges of brain matter between frontal and temporal operculum, which should normally be separated by the subarachnoid spaces of the sylvian fissure [12]. As a consequence, the virtual tumor can grow directly from one operculum to the other, resulting in unrealistic growth patterns as compared to clinically reported ones [19].

In this paper, we build an expert-revised CSF segmentation map of the MNI reference brain. We then propose to analyze how these corrections influence tumor growth patterns, by comparing simulations obtained using the native and corrected templates.

\section{Data}

The MNI 152 T1 MRI, gray matter probability map, and white matter probability map are de facto registered [9] and are all downloadable from the MNI website. The MNI 152 brain segmentation was created from the threshold gray and white matter MNI 152 probability maps (process described in following section). A neurosurgeon (A. Amelot) segmented the missing sulci patterns from the T1 image to refine this baseline brain parenchyma mask (BM), creating an improved BM. For the simulations in the Experiment section, the data inputs were the white matter segmentation (WM), which was segmented from the white matter probability map, and either the MNI 152 original brain segmentation or the improved brain segmentation (see below).

\section{Method}

In this section, we describe the preprocessing of the data and the model used to simulate tumor growth.

Preprocessing of Data: The WM, gray matter segmentation (GM), original MNI $152 \mathrm{BM}$ and improved BM were created with the following steps.

The WM and GM mark out the inhomogeneous tissue boundaries of the brain and the CSF segmentation marks out where brain tissue is not. The WM was achieved by thresholding the MNI 152 white matter probability map or a probability $>0.58$. The GM was estimated by thresholding the MNI 152 gray matter 
probability map for a probability $>0.42$ and the CSF segmentation was achieved by thresholding the MNI 152 CSF probability map for probability $>0.17$. These threshold values depict the most accurate account for where the white and gray matter and CSF are according to a neurosurgeon (E. Mandonnet).

The original MNI $152 \mathrm{BM}$ was created by combining the WM and GM segmentations and removing all voxels that were marked as CSF by the CSF segmentation.

It took several steps to enhance the original MNI 152 BM. First, we determined where the original MNI $152 \mathrm{BM}$ was imperfect by visually inspecting each slice along the three dimensions (axial, coronal, and sagittal). In this scan, we studied all sulci, gyrus, and cerebral lobes. Second, we created an improved CSF segmentation manually by redrawing, using Slicer [8], all of the subarachnoid spaces physiologically present that were lacking in the original MNI $152 \mathrm{BM}$ (Figure 1). Third, we used the improved CSF segmentation to identify additional voxels in the original MNI $152 \mathrm{BM}$ to be marked as CSF instead of brain.

The Fisher Kolmogorov (FK) Model: The Fisher Kolmogorov (FK) equation is a type of reaction-diffusion model, which has been used extensively in tumor growth modeling $[32,21,31,4,12,16,20,10,11,26,27]$. In our work, we use the following formulation:

$$
\frac{\partial u}{\partial t}=\underbrace{\nabla \cdot(D(\mathbf{x}) \nabla u)}_{\text {Diffusion Term }}+\underbrace{\rho \cdot u \cdot(1-u)}_{\text {Logistic Reaction Term }} ; \underbrace{\eta_{\partial \omega} \cdot(D \nabla u)=0}_{\text {Boundary Condition }},
$$

where $u$ is the tumor cell density, $D$ is the diffusion tensor, $\rho$ is the proliferation rate, and $\eta_{\partial \omega}$ are the normal directions to the boundaries of the brain surface.

There are several tensor construction methods that have been proposed to model anisotropic diffusion $[4,12]$. We used a tensor construction method, proposed by Clatz et al. [4], which uses global scaling on the DTI,

$$
D(\mathbf{x})= \begin{cases}d_{g} I & \text { if } \mathbf{x} \text { is in grey matter } \\ d_{w} D_{\text {water }} & \text { if } \mathbf{x} \text { is in white matter, }\end{cases}
$$

where $D(\mathbf{x})$ is the inhomogeneous diffusion term, which takes into account that tumor cells move faster along anisotropic white matter fiber tracts, estimated by $d_{w} D_{\text {water }}$, than in isotropic gray matter $d_{g} I . d_{w}$ and $d_{g}$ are scalars, $D_{\text {water }}$ is the normalized water diffusion tensor in the brain measured by the DTI, and $I$ is a $3 \times 3$ identity matrix, which can be seen as an isotropic diffusion tensor. $D_{\text {water }}$ is normalized so that the largest eigenvalue in white matter is one.

The Eikonal Approximation of the FK Model: In this section we first present the mathematical model that we considered for the description of glioma growth. This model is based on a reaction-diffusion equation (nonlinear PDE) which, even though is quite precise in predicting tumor cell density concentrations in 
the brain, it is computationally very expensive. Then, we show an approximation of this reaction-diffusion model by a traveling time formulation [14] which we use to estimate the proliferation and diffusivity parameters of the model by minimizing the mismatch between a simulated tumor and a real one extracted from MRIs.

As presented in [14] we can approximate the reaction-diffusion model in Equation 1 by considering that for large times with constant coefficients the FK model with constant coefficients admits a traveling wave solution in the infinite cylinder. In other words, when the change of $u$ is nonzero in only one direction, $\mathbf{n}$, for extremely large times the solution of Equation 1 can be formulated as

$$
u(x, t)=u(\mathbf{n} \cdot x-v t)=u(\zeta) \quad \text { as } \quad t \rightarrow \infty,
$$

where $v$ is the asymptotic speed of the wave front and $\zeta=(\mathbf{n} \cdot x-v t)$ is the moving frame of the traveling wave. Then, by substituting Equation 3 into Equation 1 and considering a constant diffusion tensor we get

$$
\mathbf{n}^{\prime} D \mathbf{n} \frac{d^{2} u}{d \zeta^{2}}+v \frac{d u}{d \zeta}+\rho u(1-u)=0,
$$

which is a non-linear ordinary differencial equation. In order to have admissible solutions, the asymptotic speed $v$ should depend on the diffusion tensor $D, \rho$, and on the shape of the initial condition $u(x, 0)$. When the initial condition has a compact support the asymptotic speed of the traveling wave can be given as, $[21]$

$$
v=2 \sqrt{\rho \mathbf{n}^{\prime} D \mathbf{n}} .
$$

However, there exist a transient behavior for the traveling wave speed while it converges to the asymptotic speed in Equation 5. Ebert et al. studied this convergence behaviour and derived in [7] the following anlaytical time-dependent representation of the traveling wave speed:

$$
v(t)=\sqrt{\mathbf{n}^{\prime} D \mathbf{n}} \frac{4 t \rho-3}{2 t \sqrt{\rho}} .
$$

Now we can formulate the traveling time formulation for the tumor deliniation. Based on the concepts described in [25], $v(t)$ can be characterized as

$$
|\nabla T|=\frac{1}{v(t)}=\left(2 \sqrt{\rho \mathbf{n}^{\prime} D \mathbf{n}}-\frac{3}{2 T} \sqrt{\frac{\mathbf{n}^{\prime} D \mathbf{n}}{\rho}}\right)^{-1},
$$

where $T$ is an implicit time function such that it embeds the locations of the tumor deliniation as iso-time surfaces $(T(x)$ is the function described as the time when the tumor delineation passes over the point $x$ ). Thus, $\mathbf{n}$ can be written as $\nabla T /|\nabla T|$ resulting in the traveling wave formulation as

$$
\sqrt{\nabla T^{T} D \nabla T}=\frac{2 \sqrt{\rho} T}{4 \rho T-3}
$$


where the equation has the Eikonal form $\left(F \sqrt{\nabla T^{T} D \nabla T}=1\right.$ with $F$ being a general speed function). This equation by itself solely gives the relation of consecutive iso-time surfaces of $T$. In order to construct the solution everywhere in the domain we require a Dirichlet type boundary condition, i.e., an initial surface for which we know the $T$ value. For the tumor growth modeling case, this surface is given by the tumor delination in the first image,i.e.,

$$
T(x)=T_{1} \quad \forall x \in \Gamma_{1},
$$

where $\Gamma_{1}$ is the tumor delination established in the first image and $T_{1}$ is the time from the origination of the tumor to the aquisition of the first image. For the asymptotic case, the traveling time formulation is given by

$$
2 \sqrt{\nabla T^{T} \rho D \nabla T}=1 .
$$

Notice from Equation 10 that $T_{1}$ is not needed for the asymptotic case and that $\rho$ and $D$ appear in the product form and cannot be separated. The formulation given in equations 8 , and 9 is valid in the infinite cylinder where the evolution is in one direction (in this situation, the traveling wave is a plane). In the case of tumor growth, the deliniation is curved and consequently its evolution is not analogous to an evolution in the infinite cylinder. We can still implement the formulation obtained in the infinite cylinder to more general cases in 3D by assuming that within a voxel, the tumor delineation is planar and the model coefficients are constant. Then by starting from the initial tumor deliniation and spweeping the domain outwards we can construct the solution. Still, such a generalization does not consider the effect of curvatue in the more general evolutions. Following the derivations of [13], [14] proposed the following Eikonal equation to describe the evolution of the tumor delineation in 3D:

$$
\left(\frac{4 \rho T-3}{2 \sqrt{\rho} T}-\nabla \cdot \frac{D \nabla T}{\sqrt{\nabla T^{T} D \nabla T}}\right) \sqrt{\nabla T^{T} D \nabla T}=1,
$$

where $\nabla \cdot\left(D \nabla T / \sqrt{\nabla T^{T} D \nabla T}\right)$ is the efect of the curvature. In the derivation of Equation 11 it is assumed that the surface is slightly curved which requires the effect of the curvature to be of a lower order than the term $2 \sqrt{\rho}$. However, the value of the curvature might be higher in the general case due to the precense of anisotropy in the diffusion process. Therefore, one needs to saturate the effect of curvature to satisfy this asumption. Thus, adding saturation to the curvature effect in Equation 11 we obtain the final formulation which describes the evolution of the tumor deliniation in 3D based on the reaction-diffusion formalism:

$$
\begin{gathered}
\left(\frac{4 \rho T-3}{2 \sqrt{\rho} T}-0.3 \sqrt{\rho}\left(1-e^{-\left|K_{e f f}\right| / 0.3 \sqrt{\rho}}\right)\right) \sqrt{\nabla T^{T} D \nabla T}=1, \\
K_{e f f}=\nabla \cdot \frac{D \nabla T}{\sqrt{\nabla T^{T} D \nabla T}}, \\
T(x)=T_{1} \quad \forall x \in \Gamma_{1},
\end{gathered}
$$


Notice that Equation 12 can become negative for low values of $T$ due to the fact that the approximation for the time convergence and curvature effects get worse for lower $T$ values [7]. In order to overcome this approximation error, [14] prevented the speed function in Equation 12 to drop lower than $0.1 \sqrt{\rho}$, i.e.,

$$
\frac{4 \rho T-3}{2 \sqrt{\rho} T}-0.3 \sqrt{\rho}\left(1-e^{-1\left|K_{e f f}\right| / 0.3 \sqrt{\rho}}\right) \geq 0.1 \sqrt{\rho},
$$

which serves as the minimum threshold for the speed of the tumor. In terms of the speed of progression of the tumor deliniation, this limit can be written as

$$
v_{\min }=0.1 \sqrt{\rho \mathbf{n}^{T} D \mathbf{n}},
$$

where $\mathbf{n}$ in the direction of the vector $\nabla T$. As a reslut of this constraint we have a growing tumor delineation at all times, consistent with the reaction-diffusion model.

\section{Experiment}

We performed a comparison between MNI original BM and improved BM using synthetic tumor simulations. Using the traveling time formulation in equations 12,13 , and 14, we simulated four synthetic tumor evolutions using both the BMs within the simulations. Each simulation was started from a voxel with a asymptotic tumor growth speed $\left(v=2 \sqrt{d_{w} \rho}\right)$ of $v=24 \mathrm{~mm} /$ year, $\rho=61 /$ year, $d_{w}=24 \mathrm{~mm}^{2} /$ year, $T_{0}=1$ day (tumor age), and $d_{g}=d_{w} / 10 \mathrm{~mm}^{2} /$ year $[14$, $29,30]$.

\section{$5 \quad$ Results}

A total of 111963 voxels have been redefined as CSF by the expert, representing $2 \%$ of the total number of brain voxels. Despite that this ratio is very small, we show now the importance of these corrections by comparing the growth patterns obtained with simulations using the baseline BM (original MNI 152) versus the improved BM for 4 different starting points.

We first selected a starting point in the deep white matter of the anterior middle frontal gyrus (see Figure 2). In this situation, there was no significant difference in the growth patterns between baseline BM and improved BM simulations.

In Figure 3, the tumor starting point was chosen in the right frontal operculum, pars triangularis of the right inferior frontal gyrus. When using the original MNI $152 \mathrm{BM}$, the tumor invaded the right insula in the first year of tumor growth, whereas the insula remained free of tumor after two years of growth when using our improved BM. This latter tumor growth pattern better depicts what is observed on a real patient (see Figure 3). Figure 4 illustrates tumor growth in the right temporal lobe. The starting point of the simulated tumor was set in the superior temporal gyrus. As seen in the coronal section 
on the original BM, the virtual tumor started to invade the middle temporal gyrus during the first year of tumor growth, as tumor cells could find a way to go through the superior temporal sulcus. The simulated tumor using the improved BM clearly shows no tumor invasion of the middle temporal gyrus and the growth remained confined to the superior temporal gyrus as observed on a real patient (see Figure 4).

\section{Discussion}

Computational modeling of glioma growth has recently emerged as an active field of biomathematics. The aim is to built a patient-specific virtual tumor, whose evolution is as close as possible to the real patient tumor. On this perfect virtual copy, several treatment sequences could be simulated, helping to select the optimal regimen. However, there is currently a huge gap between advances in numerical simulations and their effective use in clinical practice, and actually, very few studies attempted to evalute the veracity of virtual glioma growth patterns $[12,15]$.

In this paper, we showed the difference in simulated growth patterns when using two different templates of segmented CSF spaces, obtained from a baseline and an expert-revised version of the MNI 152 atlas. The mismatch between real and simulated tumors was minimal when using the expert-revised template. This result emphasizes that detailed anatomy has to be taken into account in computational models. Here, anatomical inaccuracies of sulcal anatomy in the baseline template allow virtual glioma cells to cross a sulcus - that should normally not be crossed by real glioma cells -, leading to unrealistic shapes of simulated tumors. Of course, the difference is especially great for tumors with a starting point close to important sulci. Our results reinforce the idea that glioma simulations cannot be purely be based on patient images and require anatomical information from atlases. Until advances in image analysis tools enable an automated and reliable identification of gyri and sulci, it is indeed unrealistic to perform manual segmentation of CSF spaces for each patient case. The simplest way to overcome this issue is to register all patient images in the reference space, perform the simulations in the reference space, and project the results back to the patient space. However, this approach might not always produce good results due to a possible non-correspondence between the patient and atlas geometries. The reverse method of registering the atlas onto the patient space is an alternative, but it carriers the risk of recreating artificial bridges of gray matter between the two flanks of sulci. Hence this paper has important consequences regarding the elaboration of an efficient pipeline for patient-specific tumor growth simulations. Moreover, previous works that did not make use of an expert-revised segmentation of CSF spaces could be of limited value. For example, it has been proposed to use the proliferation-diffusion model to determine the starting point of a patient glioma [16]. The starting point is indeed key information that can be used to find spatially distinct clusters of glioma [15] arising from different cells of origin, with distinct patterns of recurrence and prognosis $[17,1]$. This paper 
shows that infering tumor starting points by solving the inverse problem of the proliferation-diffusion equation is meaningless if the CSF template has not been carefully designed to take into account precise sulcal anatomy.

\section{Conclusion}

We have shown the importance of using expert-revised templates when dealing with glioma computational simulations. We invite researchers to make use of the presented map of CSF spaces in their simulations of tumor growth, or to give in the methods section how their CSF segmentation was obtained. Clinicians will not trust biomathematical models predictions unless anatomical knowledge is adequately incorporated in the modeling processes.

Acknowledgements: Erin Stretton's research work was partially funded by ERC advanced grant MedYMA.

\section{References}

1. Leif-Erik Bohman, Kristin R Swanson, Julia L Moore, Russ Rockne, Christopher Mandigo, Todd Hankinson, Marcela Assanah, Peter Canoll, and Jeffrey N Bruce. Magnetic resonance imaging characteristics of glioblastoma multiforme: implications for understanding glioma ontogeny. Neurosurgery, 67(5):1319, 2010.

2. Pierre-Yves Bondiau, Ender Konukoglu, Olivier Clatz, Herve Delingette, Marc Frenay, and Philippe Paquis. Biocomputing: Numerical simulation of glioblastoma growth and comparison with conventional irradiation margins. Physica Medica, 27(2):103-108, 2011.

3. O Clatz, M Sermesant, P-Y Bondiau, H Delingette, S K Warfield, G Malandain, and $\mathrm{N}$ Ayache. Realistic simulation of the $3-\mathrm{D}$ growth of brain tumors in MR images coupling diffusion with biomechanical deformation. IEEE Transactions on Medical Imaging, 24:1334-1346, 2005.

4. O. Clatz, M. Sermesant, P.Y. Bondiau, H. Delingette, S.K. Warfield, G. Malandain, and N. Ayache. Realistic simulation of the 3 -d growth of brain tumors in MR images coupling diffusion with biomechanical deformation. Medical Imaging, IEEE Transactions on, 24(10):1334-1346, 2005.

5. David Corwin, Clay Holdsworth, Russell C Rockne, Andrew D Trister, Maciej M Mrugala, Jason K Rockhill, Robert D Stewart, Mark Phillips, and Kristin R Swanson. Toward patient-specific, biologically optimized radiation therapy plans for the treatment of glioblastoma. PloS one, 8(11):e79115, 2013.

6. G Cruywagen, D Woodward, P Tracqui, G Bartoo, J Murray, and E. Alvord. The modelling of diffusive tumours. j biol systems. J Biol Systems, 3:937-45, 1995.

7. Ute Ebert and Wim van Saarloos. Front propagation into unstable states: universal algebraic convergence towards uniformly translating pulled fronts. Physica D: Nonlinear Phenomena, 146(1):1-99, 2000.

8. Andriy Fedorov, Reinhard Beichel, Jayashree Kalpathy-Cramer, Julien Finet, Jean-Christophe Fillion-Robin, Sonia Pujol, Christian Bauer, Dominique Jennings, Fiona Fennessy, Milan Sonka, et al. 3d slicer as an image computing platform for the quantitative imaging network. Magnetic Resonance Imaging, 2012. 
9. VS Fonov, AC Evans, RC McKinstry, CR Almli, and DL Collins. Unbiased nonlinear average age-appropriate brain templates from birth to adulthood. Neuroimage, 47:S102-S102, 2009.

10. A. Gooya, G. Biros, and C. Davatzikos. Deformable registration of glioma images using em algorithm and diffusion reaction modeling. IEEE Trans Med Imaging, 30(2):375-390, Feb 2011.

11. C. Hogea, C. Davatzikos, and G. Biros. An image-driven parameter estimation problem for a reaction-diffusion glioma growth model with mass effects. J. of Math. Bio., 56(6):793-825, 2008.

12. S. Jbabdi, E. Mandonnet, H. Duffau, L. Capelle, K. Swanson, M. Pélégrini-Issac, R. Guillevin, and H. Benali. Simulation of anisotropic growth of low-grade gliomas using diffusion tensor imaging. Magnetic Resonance in Medicine, 54(3):616-624, 2005.

13. James Keener and James Sneyd. Mathematical physiology, interdisciplinary applied mathematics 8, 1998.

14. E. Konukoglu. Modeling Glioma Growth and Personalizing Growth Models in Medical Images. PhD thesis, University of Nice, 2009.

15. E. Konukoglu, O. Clatz, P.Y. Bondiau, H. Delingette, and N. Ayache. Extrapolating glioma invasion margin in brain magnetic resonance images: Suggesting new irradiation margins. Medical image analysis, 14(2):111-125, 2010.

16. E. Konukoglu, O. Clatz, B. Menze, B. Stieltjes, M. Weber, E. Mandonnet, H. Delingette, and N. Ayache. Image guided personalization of reaction-diffusion type tumor growth models using modified anisotropic eikonal equations. Medical Imaging, IEEE Transactions on, 29(1):77-95, 2009.

17. Daniel A Lim, Soonmee Cha, Mary C Mayo, Mei-Hsiu Chen, Evren Keles, Scott VandenBerg, and Mitchel S Berger. Relationship of glioblastoma multiforme to neural stem cell regions predicts invasive and multifocal tumor phenotype. Neurooncology, 9(4):424-429, 2007.

18. E Mandonnet. Mathematical modeling of glioma on mri. Revue neurologique, 167(10):715-720, 2011.

19. Emmanuel Mandonnet, Laurent Capelle, and Hugues Duffau. Extension of paralimbic low grade gliomas: toward an anatomical classification based on white matter invasion patterns. Journal of neuro-oncology, 78(2):179-185, 2006.

20. B. H. Menze, E. Stretton, E. Konukoglu, and N. Ayache. Image-based modeling of tumor growth in patients with glioma. Springer, Heidelberg/Germany, 2011.

21. J.D. Murray. Mathematical biology, volume 2. Springer, 2002.

22. Maxwell Lewis Neal, Andrew D Trister, Tyler Cloke, Rita Sodt, Sunyoung Ahn, Anne L Baldock, Carly A Bridge, Albert Lai, Timothy F Cloughesy, Maciej M Mrugala, et al. Discriminating survival outcomes in patients with glioblastoma using a simulation-based, patient-specific response metric. PloS one, 8(1):e51951, 2013.

23. Benjamin Ribba, Gentian Kaloshi, Mathieu Peyre, Damien Ricard, Vincent Calvez, Michel Tod, Branka Čajavec-Bernard, Ahmed Idbaih, Dimitri Psimaras, Linda Dainese, et al. A tumor growth inhibition model for low-grade glioma treated with chemotherapy or radiotherapy. Clinical Cancer Research, 18(18):5071-5080, 2012.

24. R Rockne, EC Alvord Jr, JK Rockhill, and KR Swanson. A mathematical model for brain tumor response to radiation therapy. Journal of mathematical biology, 58(4-5):561-578, 2009.

25. James Albert Sethian. Level set methods and fast marching methods: evolving interfaces in computational geometry, fluid mechanics, computer vision, and materials science, volume 3. Cambridge university press, 1999. 
26. E Stretton, E Geremia, B Menze, H Delingette, and N Ayache. Importance of patient DTI's to accurately model glioma growth using the reaction diffusion equation. ISBI, 2013.

27. E. Stretton, E. Mandonnet, E. Geremia, B.H. Menze, H. Delingette, and N. Ayache. Predicting the location of glioma recurrence after a resection surgery. Medical Image Computing and Computer-Assisted Intervention, MICCAI 2012, 2012.

28. K. Swanson, E. Alvord, and J. Murray. A quantitative model for differential motility of gliomas in grey and white matter. Cell Proliferation, 33(5):317-330, 2000.

29. K. Swanson, C. Bridge, J. Murray, and E. Alvord. Virtual and real brain tumors: using mathematical modeling to quantify glioma growth and invasion. Journal of the neurological sciences, 216(1):1-10, 2003.

30. K.R. Swanson. Mathematical Modeling of the Growth and Control of Tumors. PhD thesis, University of Washington, 1999.

31. KR Swanson, RC Rostomily, and EC Alvord. A mathematical modelling tool for predicting survival of individual patients following resection of glioblastoma: a proof of principle. British journal of cancer, 98(1):113-119, 2007.

32. P. Tracqui, G. Cruywagen, D. Woodward, G. Bartoo, and J. MurrayE.C. Alvord Jr. A mathematical model of glioma growth: the effect of chemotherapy on spatiotemporal growth. Cell Proliferation, 28(1):17-31, 1995.

33. P Tracqui, G C Cruywagen, D E Woodward, G T Bartoo, J D Murray, and E C Alvord. A mathematical model of glioma growth: the effect of chemotherapy on spatio-temporal growth. Cell Prolif, 28:17-31, 1995.

34. Christina H Wang, Jason K Rockhill, Maciej Mrugala, Danielle L Peacock, Albert Lai, Katy Jusenius, Joanna M Wardlaw, Timothy Cloughesy, Alexander M Spence, Russ Rockne, et al. Prognostic significance of growth kinetics in newly diagnosed glioblastomas revealed by combining serial imaging with a novel biomathematical model. Cancer research, 69(23):9133-9140, 2009.

35. DE Woodward, J Cook, P Tracqui, GC Cruywagen, JD Murray, and EC Alvord. A mathematical model of glioma growth: the effect of extent of surgical resection. Cell proliferation, 29(6):269-288, 1996. 


\section{Compare BMs}

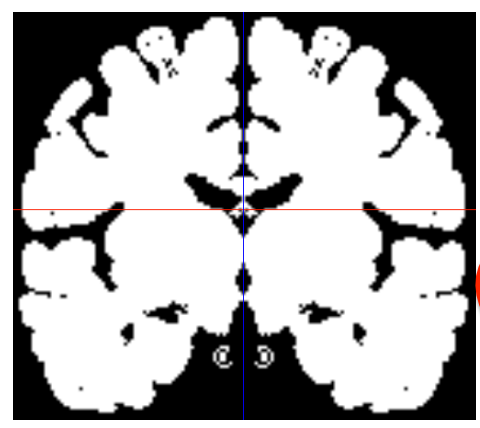

MNI Original BM

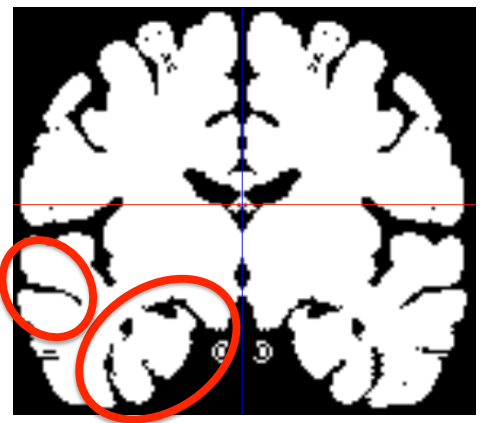

Corrected BM

Fig. 1: MNI 152 original BM vs improved BM. Left, the MNI 152 original BM is displayed. Right, the improved BM is shown with the changes highlighted by red circles.

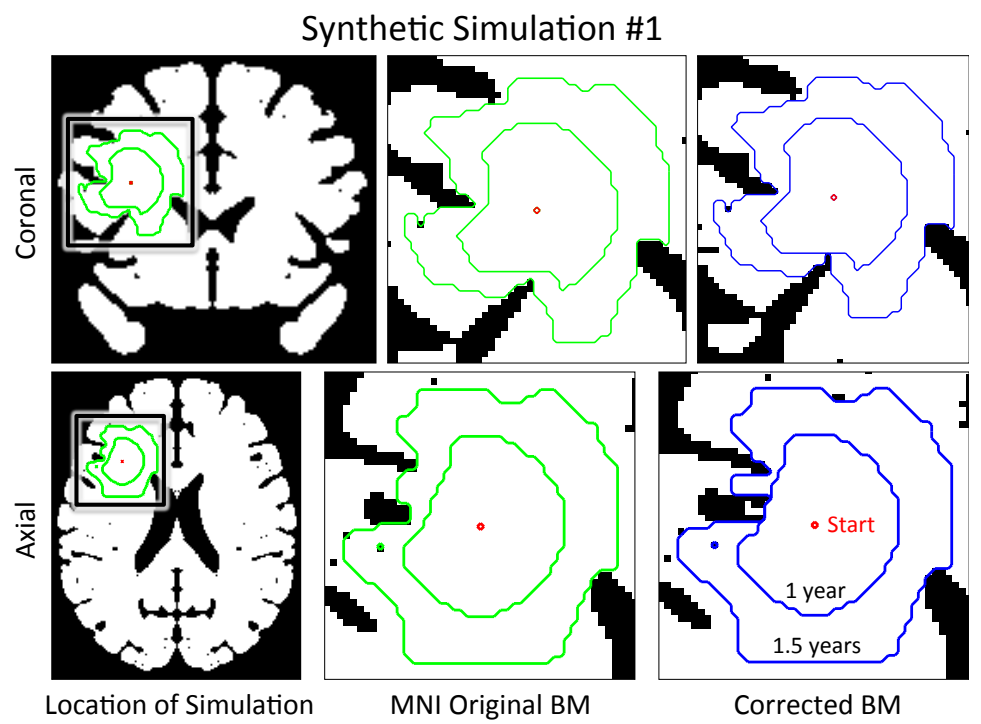

Fig. 2: Comparison between MNI 152 original BM and improved BM using synthetic tumor simulation \#1. The BMs are the background of each image. Left images show the location in the BM where this synthetic tumor was simulated. On the right bottom is displayed a time map (shown for 1 and 1.5 years) for this synthetic tumor that was started from a single voxel (red). Notice that the simulations run using the original MNI $152 \mathrm{BM}$ and improved MNI $152 \mathrm{BM}$ are the same for a tumor growing in the white matter of anterior middle frontal gyrus (2 cm lateral to the frontal horn). 


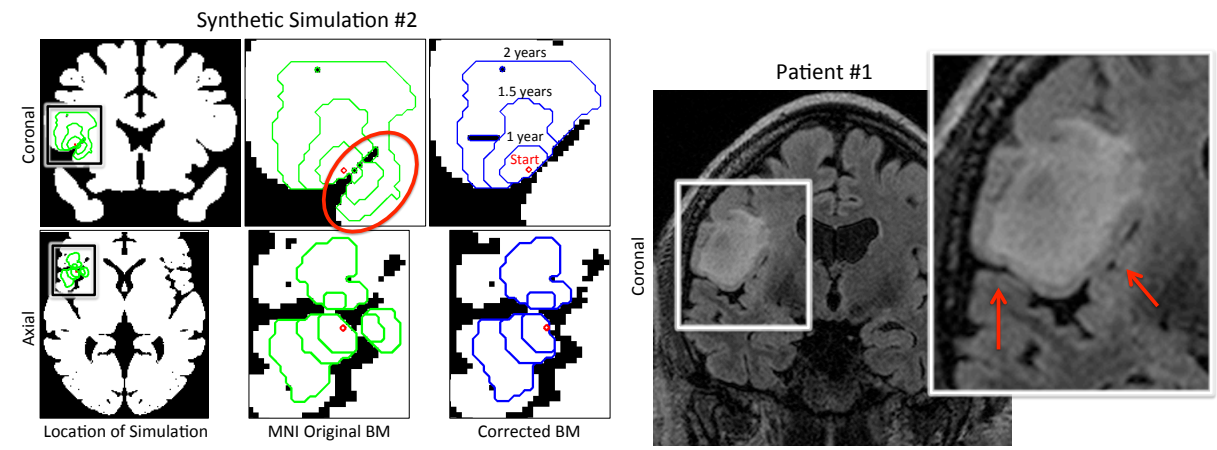

Fig. 3: Left: Comparison between MNI 152 original BM and improved BM using synthetic tumor simulation \#2. The BMs are the background of each image. Left images show the location in the BM where this synthetic tumor was simulated. On the right top is displayed a time map (shown for $1,1.5$, and 2 years) for this synthetic tumor that was started from a single voxel (red). A red circle is drawn around an area where the synthetic tumor grew because the MNI 152 original BM (background) was incorrect. Right is displayed the simulation which used the improved BM where this synthetic tumor did not grow across the sylvian fissure incorrectly. Right: Patient \#1 had a tumor in the same place as where the synthetic simulation \#2 was grown. This demonstrates the importance of the improved BM as there was indeed no tumor growth into the insular and superior temporal gyrus (red arrows). 


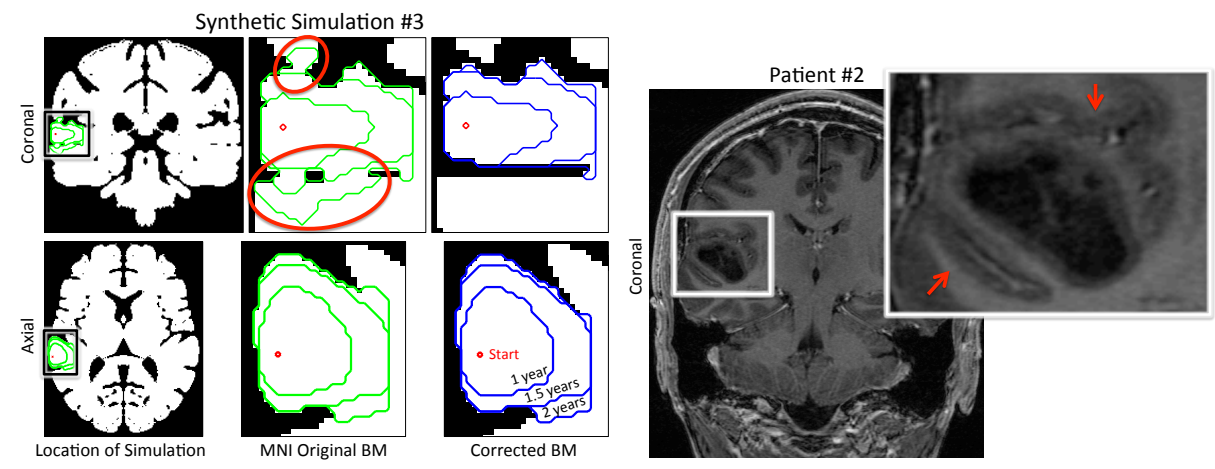

Fig. 4: Left: Comparison between MNI 152 original BM and improved BM using synthetic tumor simulation \#3. The BMs are the background of each image. Left images show the location in the BM where this synthetic tumor was simulated. On the right bottom is displayed a time map (shown for 1, 1.5, and 2 years) for this synthetic tumor that was started from a single voxel (red). A red circle is drawn around an area where the synthetic tumor grew because the MNI 152 original BM (background) was incorrect. Right is displayed the simulation which used the improved BM where the synthetic tumor did not grow across the sulci incorrectly. Right: Patient \#2 had a tumor in the same place as where the synthetic simulation \#3 was grown. This demonstrates the importance of the improved BM in the superior temporal gyrus, as the tumor growth remained confined to the superior temporal gyrus, with no growth across the superior temporal sulcus and sylvan fissure (red arrows). 